\title{
Culture in Reduced Levels of Oxygen Promotes Clonogenic Sympathoadrenal Differentiation by Isolated Neural Crest Stem Cells
}

\author{
Sean J. Morrison, ${ }^{1,3}$ Marie Csete, ${ }^{2}$ Andrew K. Groves, ${ }^{4}$ William Melega, ${ }^{5}$ Barbara Wold, ${ }^{2}$ and \\ David J. Anderson ${ }^{1,2}$ \\ ${ }^{1}$ Howard Hughes Medical Institute and 2Division of Biology, California Institute of Technology, Pasadena, California 91125, \\ ${ }^{3}$ Departments of Internal Medicine and Cell and Developmental Biology, University of Michigan, Ann Arbor, Michigan \\ 48109-0934, 4Department of Cell and Molecular Biology, House Ear Institute, Los Angeles, California 90057, and \\ ${ }^{5}$ Department of Pharmacology, University of California Los Angeles, Los Angeles, California 90095
}

Isolated neural crest stem cells (NCSCs) differentiate to autonomic neurons in response to bone morphogenetic protein 2 (BMP2) in clonal cultures, but these neurons do not express sympathoadrenal (SA) lineage markers. Whether this reflects a developmental restriction in NCSCs or simply inappropriate culture conditions was not clear. We tested the growth and differentiation potential of NCSCs at $\sim 5 \% \mathrm{O}_{2}$, which more closely approximates physiological oxygen levels. Eighty-three percent of $\mathrm{p} 75^{+} \mathrm{P}_{0}{ }^{-}$cells isolated from embryonic day 14.5 sciatic nerve behaved as stem cells under these conditions, suggesting that this is a nearly pure population. Furthermore, addition of BMP2 plus forskolin in decreased oxygen cultures elicited differentiation of thousands of cells expressing tyrosine hydroxylase, dopamine- $\beta$-hydroxylase, and the SA lineage marker SA-1 in nearly all colonies. Such cells also synthesized and released dopamine and norepinephrine. These data demonstrate that isolated mammalian NCSCs uniformly possess SA lineage capacity and further suggest that oxygen levels can influence cell fate. Parallel results indicating that reduced oxygen levels can also promote the survival, proliferation, and catecholaminergic differentiation of CNS stem cells (Studer et al., 2000) suggests that neural stem cells may exhibit a conserved response to reduced oxygen levels.

Key words: sympathoadrenal; neural crest; stem cell; hypoxia; oxygen; sympathetic neuron; dopamine; noradrenergic; autonomic differentiation; cell fate determination
Catecholaminergic (dopaminergic, noradrenergic, and adrenergic) derivatives of the peripheral nervous system (PNS) are derived from the sympathoadrenal (SA) lineage of the neural crest. SA derivatives include sympathetic neurons, adrenal chromaffin cells, carotid body cells, and small intensely fluorescent (SIF) cells (Doupe et al., 1985a,b; Anderson, 1993). Although multipotent neural crest progenitors have been observed to give rise to SA derivatives in vivo (Fraser and Bronner-Fraser, 1991), relatively little is known about the mechanisms underlying the commitment of such cells to the SA sublineage. Bone morphogenetic proteins (BMPs) have been identified as inducers of SA marker expression in mass cultures of avian and mammalian neural crest cells (Varley et al., 1995; Reissman et al., 1996; Varley and Maxwell, 1996; Lo et al., 1999). Such BMPs can also induce autonomic neuron differentiation in clonal cultures of mammalian neural crest stem cells (NCSCs) (Stemple and Anderson, 1992; Shah et al., 1996), but they do not induce SA lineage markers. It is not clear whether this reflects a restriction in the developmental potential of NCSCs or, rather, a requirement for additional signals besides BMPs and forskolin to elicit SA differentiation at clonal density.

The development of catecholaminergic cells of the SA lineage is not only of basic interest but is also relevant to human disease. Parkinson's disease is a relatively common neurodegenerative disorder caused by the loss of dopaminergic neurons in the substantia

\footnotetext{
Received May 12, 2000; revised July 5, 2000; accepted July 6, 2000.

This work was supported by a program project grant from the National Institutes of Health (B.W., Principle Investigator). S.J.M. was initially supported by a postdoctoral fellowship from the American Cancer Society, California Division and is currently an Assistant Investigator of the Howard Hughes Medical Institute. D.J.A. is an Investigator of the Howard Hughes Medical Institute. We thank Gaby Mosconi for laboratory management, Suzanne Bixby, Lan Dinh, Hieu Phan, and Ling Wang for technical assistance, and Robert Vega for animal ordering. We thank Rochelle Diamond and Pat Koen of the Caltech Flow-Cytometry Facility for FACS operation and J. J. Archelos for monoclonal anti- $\mathrm{P}_{0}$ antibody.

Correspondence should be addressed to David J. Anderson, Howard Hughes Medical Institute, California Institute of Technology, Pasadena, CA 91125. E-mail: mancosog@cco.caltech.edu.

Copyright (C) 2000 Society for Neuroscience $0270-6474 / 00 / 207370-07 \$ 15.00 / 0$
}

nigra. The transplantation of a wide variety of dopaminergic cell types, including fetal mesencephalon (Freed et al., 1992) and carotid body cells (Luquin et al., 1999), into the substantia nigra can ameliorate the symptoms of Parkinson's disease; however, the supply of fetal tissue is very limited (Bjorklund, 1993). Extensive efforts have been made to identify alternate sources of dopaminergic neurons (Zawada et al., 1998) and to expand dopaminergic precursor cells in culture (Studer et al., 1998). Encouragingly, transplantation of dopaminergic cells derived from cultured CNS fetal progenitors has been shown to alleviate Parkinson-like symptoms in rats (Studer et al., 1998). However, dopaminergic differentiation by neural progenitors in culture has tended to be inefficient.

We recently developed a procedure to isolate $\mathrm{p} 75^{+} \mathrm{P}_{0}{ }^{-}$postmigratory NCSCs from uncultured rat sciatic nerve by flow cytometry (Morrison et al., 1999). Like NCSCs derived from neural tube explant cultures (Stemple and Anderson, 1992), these cells selfrenew and give rise to autonomic neurons and glia in vivo and in vitro. Previous studies of NCSC differentiation in culture have been performed in $20 \%$ oxygen (from $94 \%$ air and $6 \%$ carbon dioxide) as is standard in most tissue culture experiments. However, mean tissue oxygen levels in vivo are only 3\% (Guyton and Hall, 1996). To generate a more physiological environment, we cultured NCSCs in decreased oxygen levels (3-6\% oxygen). Decreased oxygen levels promoted the survival, proliferation, and multilineage differentiation of NCSCs, indicating that the $\mathrm{p} 75^{+} \mathrm{P}_{0}{ }^{-}$postmigratory NCSC population (Morrison et al., 1999) is $80-95 \%$ pure for NCSCs. In decreased oxygen cultures, addition of BMP2 plus forskolin caused virtually all $\mathrm{p} 75^{+} \mathrm{P}_{0}{ }^{-} \mathrm{NCSC}$ s to generate colonies containing SA lineage cells. The identification of culture conditions that permit individual, purified NCSCs to produce thousands of catecholaminergic cells will greatly facilitate further studies of SA lineage commitment and may also provide a source of cells for transplantation into animal models of Parkinson's disease. Similarly, Studer et al. (2000) (companion article) have observed that decreased oxygen culture promotes the survival, proliferation, and dopaminergic differentiation of CNS stem 
Table 1. Culture of sciatic nerve $\mathrm{p}^{+} \mathrm{P}_{0}^{-}$cells in decreased oxygen promotes neural crest stem cell survival and multilineage differentiation

\begin{tabular}{|c|c|c|c|c|c|c|}
\hline & \multirow{2}{*}{$\begin{array}{l}\text { Plating effi- } \\
\text { ciency }(\%)\end{array}$} & \multicolumn{5}{|c|}{ Frequency of colony types (\%) } \\
\hline & & $\mathrm{N}+\mathrm{S}+\mathrm{M}$ & $\mathrm{N}+\mathrm{S}$ & $\mathrm{S}+\mathrm{M}$ & S only & M only \\
\hline $20 \% \mathrm{O}_{2}$ & $35.0 \pm 9.1$ & $48.0 \pm 16.4$ & $1.0 \pm 2.1$ & $4.8 \pm 3.4$ & $43.7 \pm 18.5$ & $2.5 \pm 3.0$ \\
\hline$\sim 5 \% \mathrm{O}_{2}$ & $48.0 \pm 5.8^{*}$ & $82.8 \pm 12.0^{*}$ & $0.4 \pm 1.0$ & $8.1 \pm 7.4$ & $6.2 \pm 3.6^{*}$ & $2.5 \pm 3.1$ \\
\hline
\end{tabular}

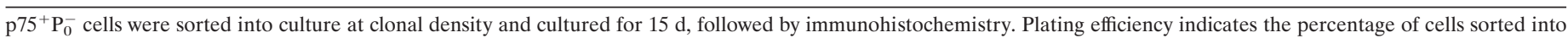

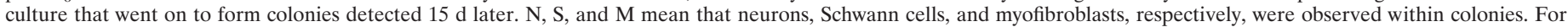

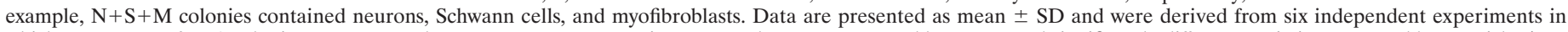

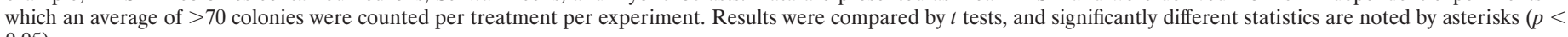
$0.05)$.

cells. Together, these results suggest that oxygen levels can enhance catecholaminergic differentiation by neural stem cells from both the CNS and PNS.

\section{MATERIALS AND METHODS}

Isolation of sciatic nerve $p 75^{+} P_{o}{ }^{-}$cells. Pregnant Sprague Dawley rats were obtained from Simonsen (Gilroy, CA). For timed pregnancies, animals were put together in the afternoon, and the morning on which the plug was observed was designated embryonic day 0.5 (E0.5). NCSCs were isolated from E14.5 sciatic nerves as described previously (Morrison et al., 1999). Briefly, nerves were dissected and dissociated by incubating in trypsin plus collagenase for $4 \mathrm{~min}$ at $37^{\circ} \mathrm{C}$, followed by mechanical trituration. The cells were stained with monoclonal antibodies against p75 (192Ig), the lowaffinity neurotrophin receptor, and $\mathrm{P}_{0}(\mathrm{P} 07)$, a PNS myelin component. p $75^{+} \mathrm{P}_{0}{ }^{-}$cells were sorted using a FACSVantage flow-cytometer (BectonDickinson, San Jose, CA).

Culture conditions. Sciatic nerve progenitors were cultured at clonal density as described previously (Morrison et al., 1999). Cells were cultured at clonal density so that the differentiation of individual NCSCs could be monitored. Clonal density means that NCSCs were plated at a very low density; typically, $40 \mathrm{NCSCs}$ were added per $35 \mathrm{~mm}$ dish, resulting in the formation of $10-20$ colonies. At this density, individual colonies were still well separated, even after proliferating for 2-3 weeks. This allowed the lineage compositions of individual clones to be determined (by immunofluorescence staining of lineage markers) and compared. Plates were coated with poly-D-lysine (Biomedical Technologies, Stoughton, MA) and human fibronectin (Biomedical Technologies). The culture medium contained DMEM-low glucose (Life Technologies, Gaithersburg, MD) with $15 \%$ chick embryo extract (CEE) (Stemple and Anderson, 1992), $20 \mathrm{ng} / \mathrm{ml}$ recombinant human basic FGF (bFGF) (R\&D Systems, Minneapolis, MN), $1 \%$ N2 supplement (Life Technologies), 2\% B27 supplement (Life Technologies), $50 \mu \mathrm{M}$ 2-mercaptoethanol, $35 \mathrm{ng} / \mathrm{ml}$ retinoic acid (Sigma, St. Louis, MO), and penicillin-streptomycin (BioWhittaker, Walkersville, MD). This composition is described throughout as "standard medium." Under standard conditions, cells were cultured for $6 \mathrm{~d}$ in standard medium and then switched to a similar medium (with $1 \% \mathrm{CEE}$ and $10 \mathrm{ng} / \mathrm{ml} \mathrm{bFGF}$ ) that favors differentiation for another $8 \mathrm{~d}$ before immunohistochemical analysis of colony composition. To promote sympathetic differentiation, 5 $\mu \mathrm{M}$ forskolin and $1 \mathrm{ng} / \mathrm{ml} \mathrm{BMP} 2$ (Genetics Institute, Cambridge, MA) were added after the first $6 \mathrm{~d}$ of standard culture, and the cultures were allowed to develop for another $6 \mathrm{~d}$. To further promote sympathetic neuron differentiation, the cultures were switched to standard medium containing $50 \mathrm{ng} / \mathrm{ml} \mathrm{NGF}$ and $50 \mathrm{ng} / \mathrm{ml}$ neurotrophin 3 (NT-3) for a final $6 \mathrm{~d}$ (after $6 \mathrm{~d}$ in standard medium, followed by $6 \mathrm{~d}$ in BMP2 plus forskolin).

Normal humidified tissue culture incubators with $6 \% \mathrm{CO}_{2}$ were used for the $20 \%$ oxygen cultures. For decreased oxygen cultures, plates were inserted into gas-tight modular incubator chambers (Billups-Rothenberg, Del Mar, CA) that were flushed with a custom gas mixture containing $1 \%$ $\mathrm{O}_{2} / 6 \% \mathrm{CO}_{2} /$ balance $\mathrm{N}_{2}$. The incubator chambers were flushed for $1.5-2$ min daily at a rate of $15 \mathrm{l} / \mathrm{min}$ and then inserted into normal tissue culture incubators. This achieved an actual concentration inside the chamber of $3-6 \%$ oxygen, based on direct measurement with a microelectrode (Animus Corp., Malvern, PA). Once cultures were established in the reduced oxygen chambers, opening of the gas-tight chambers was minimized to avoid reperfusion injury upon exposure to air (nonetheless, these cultures were refed with new media every $6 \mathrm{~d}$ according to the schedule described above).

Immunohistochemistry. For routine analysis of culture compositions, cultures were fixed in acid ethanol for $20 \mathrm{~min}$ and stained with antibodies against peripherin (AB1530; Chemicon, Temecula, CA), smooth muscle actin (SMA) (A-2547; Sigma), and glial fibrillary acidic protein (GFAP) (G-3893; Sigma) as described previously (Morrison et al., 1999). To stain for sympathetic markers, cultures were fixed in $4 \%$ paraformaldehyde for $10 \mathrm{~min}$, blocked in PBS with $4 \%$ goat serum, $0.2 \% \mathrm{BSA}$, and $0.1 \% \mathrm{NP}-40$ for $15 \mathrm{~min}$, and then stained with antibodies against tyrosine hydroxylase (TH) (Boehringer Mannheim, Indianapolis, IN), dopamine- $\beta$-hydroxylase (DBH) (PharMingen, San Diego, CA), or SA-1 (a gift of Paul Patterson, California Institute of Technology, Pasadena, CA) at room temperature for $2 \mathrm{hr}$.
HPLC analysis of dopamine and norepinephrine. A Shimadzu solvent delivery system was used with the following mobile phase: $92 \% 75 \mathrm{~mm}$ $\mathrm{NaH}_{2} \mathrm{PO}_{4}, 1.7 \mathrm{~mm}$ octanesulfonic acid, $0.05 \mathrm{~mm}$ EDTA, pH 3.1, and $8 \%$ acetonitrile, at a flow rate of $0.8 \mathrm{ml} / \mathrm{min}$. An Alltech (Deerfield, IL) Absorbosphere HS C18 reverse phase column $(10 \times 4.6 \mathrm{~mm}, 3 \mu \mathrm{m})$ was connected to an electrochemical detector (Coulochem II; ESA Inc., Chelmsford, MA) set at an applied potential of $+0.02 \mathrm{~V}$ at detector 1 and $+0.40 \mathrm{~V}$ at detector 2. Detector response was linear for $0.05-10 \mathrm{ng}(r=$ 0.99 for linear regression calculations of all compounds assayed; withinassay variance was $<5 \%$ ). Peak areas were quantitated with a Rainin (Ridgefield, NJ) MACintegrator system. No dopamine (DA) or norepinephrine (NE) was detected in blank samples prepared from solvent or from a control colony cultured under standard conditions in $20 \%$ oxygen.

\section{RESULTS}

\section{Decreased oxygen culture promotes the survival, proliferation, and neuronal differentiation of NCSCs}

Some of us (M. Csete and B. Wold) observed that the survival and proliferation of a variety of progenitor cell types in culture can be enhanced by decreasing oxygen from $20 \%$ to more physiological levels $(3-6 \%)$. We decided to test whether the survival and proliferation of NCSCs was also enhanced in reduced oxygen levels. NCSCs were isolated by flow-cytometry from the sciatic nerves of E14.5 rats and sorted into culture at clonal density under standard conditions (Morrison et al., 1999). Some cultures were kept in normal incubators containing $6 \% \mathrm{CO}_{2}$ and $20 \% \mathrm{O}_{2}$ (from air), whereas other cultures were kept in gas-tight chambers that were flushed with $1 \% \quad \mathrm{O}_{2} / 6 \% \mathrm{CO}_{2} /$ balance $\mathrm{N}_{2}$ to generate actual $\mathrm{O}_{2}$ levels of $3-6 \%$. The gas-tight chambers were housed inside the normal incubators that also contained the control cultures. After $15 \mathrm{~d}$, the plates were stained with antibodies against peripherin (to detect neurons), GFAP (to detect glia), and SMA (to detect myofibroblasts). The results are presented in Table 1 . The ability of p $75^{+} \mathrm{P}_{0}{ }^{-}$cells to survive and form colonies was significantly greater in decreased oxygen; $35 \%$ of cells added to culture formed colonies in $20 \%$ oxygen versus $48 \%$ of cells in decreased oxygen $(p<0.01)$.

In addition to improved survival, $\mathrm{p} 75^{+} \mathrm{P}_{0}{ }^{-}$cells were more likely to form multilineage colonies in decreased oxygen. In $20 \%$ oxygen, only $48 \%$ of colonies were multilineage (containing neurons, Schwann cells, and myofibroblasts) (Table $1, \mathrm{~N}+\mathrm{S}+\mathrm{M}$ ), with the balance being mainly Schwann-only colonies (Table $1, \mathrm{~S}$ only). In contrast, in decreased oxygen cultures, significantly more colonies were multipotent $(82 \%)$ and significantly fewer colonies were Schwann-only (6\%). We tested whether decreased oxygen levels were toxic to restricted progenitors by culturing E18.5 sciatic nerve cells in standard medium in either decreased or $20 \%$ oxygen. Multipotent progenitors have not been detected in the sciatic nerve after E17.5, so most or all neural progenitors in the E18.5 nerve appear to be glial committed (Jessen and Mirsky, 1999; Morrison et al., 1999). The frequencies of Schwann-only or myofibroblast-only colonies did not differ between decreased (3-6\%) and standard (20\%) oxygen cultures; $23 \%$ of E18.5 sciatic nerve cells formed Schwann-only colonies, and $22 \%$ formed myofibroblast-only colonies, irrespective of oxygen level (the remaining $55 \%$ of cells died without forming colonies in both treatments). Consistent with this, decreased oxygen did not affect the overall plating efficiency in mass cultures of E14.5 sciatic nerve cells, of which NCSCs consti- 
Table 2. BMP2 instructed neuronal differentiation of sciatic nerve $\mathrm{p}^{+} \mathrm{P}_{0}^{-}$cells is promoted by culture in decreased oxygen

\begin{tabular}{llllr} 
& & \multicolumn{2}{l}{ Types of colonies $(\%)$} \\
\cline { 4 - 5 } & Plating efficiency $(\%)$ & All neurons & Some neurons & No neurons \\
\hline No add & & & & \\
$20 \% \mathrm{O}_{2}$ & $22.7 \pm 8.5$ & $0.0 \pm 0.0$ & $0.0 \pm 0.0$ & $100.0 \pm 0.0$ \\
$\sim 5 \% \mathrm{O}_{2}$ & $33.4 \pm 7.3^{*}$ & $0.0 \pm 0.0$ & $0.4 \pm 0.9$ & $99.6 \pm 0.9$ \\
$+\mathrm{BMP} 2$ & & & & \\
$20 \% \mathrm{O}_{2}$ & $26.8 \pm 8.9$ & $31.5 \pm 17.9$ & $52.5 \pm 18.9$ & $15.9 \pm 5.7$ \\
$\sim 5 \% \mathrm{O}_{2}$ & $43.4 \pm 11.5^{*}$ & $59.5 \pm 10.8^{*}$ & $35.0 \pm 11.1$ & $5.5 \pm 1.4^{*}$
\end{tabular}

p75 ${ }^{+} \mathrm{P}_{0}^{-}$cells were sorted into cultures at clonal density and cultured for 5 or $6 \mathrm{~d}$, followed by staining for peripherin, a marker of peripheral neurons. Plating efficiency indicates the percentage of cells initially sorted into culture that went on to form colonies. Data are presented as mean \pm SD for five independent experiments. Results at different oxygen concentrations (within no add or +BMP2 treatments) were compared by $t$ tests, and significantly different statistics are noted by asterisks $(p<0.05)$. Decreased oxygen culture significantly increased plating efficiency in both treatments and neuronal differentiation in the presence of BMP2.

tuted only $15-20 \%$ (data not shown). These data support the idea that decreased oxygen culture promotes the survival and multilineage differentiation of NCSCs, without biasing against the survival of more restricted progenitors. We cannot rule out the possibility that the $52 \%$ of $\mathrm{p}^{+} 5^{+} \mathrm{P}_{0}{ }^{-}$cells that did not form colonies in culture were less enriched for NCSCs than the $48 \%$ of $\mathrm{p}^{2} 5^{+} \mathrm{P}_{0}{ }^{-}$cells that did form colonies; however, given that all known classes of progenitors from the sciatic nerve form colonies under these culture conditions (Morrison et al., 1999), the data suggest that the $\mathrm{p} 75^{+} \mathrm{P}_{0}{ }^{-}$population contains $>80 \%$ NCSCs.

To confirm that the increase in multilineage differentiation was attributable to reduced oxygen levels rather than some other aspect of culture in the gas-tight chambers, an experiment was performed in which some cultures of unseparated sciatic nerve cells were incubated in a normal incubator $\left(6 \% \mathrm{CO}_{2}\right.$ and $20 \% \mathrm{O}_{2}$ from air), whereas other cultures were incubated in gas-tight chambers that were flushed either with $1 \% \mathrm{O}_{2} / 6 \% \mathrm{CO}_{2} /$ balance $\mathrm{N}_{2}$ or $5 \% \mathrm{O}_{2} / 6 \%$ $\mathrm{CO}_{2} /$ balance $\mathrm{N}_{2}$. Although the cultures in the chamber flushed with $5 \%$ oxygen had a similar frequency of multipotent clones as the cultures in the normal incubator, the cultures in the chamber flushed with $1 \%$ oxygen had almost twice as many multipotent colonies. Because the only difference between the chambers was that those flushed with $5 \%$ oxygen would have a higher final oxygen concentration than those flushed with $1 \%$ oxygen, this result suggests that it is the lower oxygen concentration that promoted multilineage differentiation rather than some other aspect of culture in the gas-tight chamber.

To test whether $\mathrm{p} 75^{+} \mathrm{P}_{0}{ }^{-}$cells self-renew in decreased oxygen culture, a single cell was deposited per well of a 96 well plate, and then seven colonies were subcloned after $7 \mathrm{~d}$. All founder clones gave rise to multipotent subclones, averaging $158 \pm 149$ multipotent subclones per founder. These results demonstrate that individual $\mathrm{p} 75^{+} \mathrm{P}_{0}{ }^{-}$cells self-renew in decreased oxygen, as was previously documented in $20 \%$ oxygen (Morrison et al., 1999).

Decreased oxygen culture also promoted proliferation. $\mathrm{p} 75^{+} \mathrm{P}_{0}{ }^{-}$ cells were cultured at either $20 \%$ or decreased oxygen and then stained daily with $4^{\prime}, 6^{\prime}$-diamidino-2-phenylindole (DAPI) to count the total number of cells per colony and the number of dead cells per colony. On days 1 and 2 of culture, the number of cells per colony was similar in both treatments, but at later time points, colonies cultured at decreased oxygen always averaged more cells. Dead cells always averaged $<10 \%$ of cells per colony, and at most time points most colonies did not contain any dead cells. Colonies cultured in decreased oxygen did not have significantly fewer dead cells at any time point, and in fact averaged more dead cells per colony on days 5 and 6 . For example, after $4 \mathrm{~d}$ of culture colonies averaged $12 \pm 4$ and $21 \pm 3$ cells in $20 \%$ and decreased oxygen, respectively, but with only 0.7 dead cells per colony in both treatments. After $6 \mathrm{~d}$ of culture, colonies in $20 \%$ and decreased oxygen averaged $60 \pm 21$ and $132 \pm 36$ cells per colony, with 0.4 and 2.6 dead cells per colony, respectively. These data suggest that $\mathrm{p} 75^{+} \mathrm{P}_{0}{ }^{-}$progenitors proliferate more quickly in decreased oxygen culture.

Because a higher proportion of colonies fail to undergo multilineage differentiation at $20 \%$ oxygen (Table 1 ) and therefore form much smaller colonies, we wondered whether there was a difference in proliferation even when the analysis was restricted to clones undergoing multilineage differentiation. As described previously (Morrison et al., 1999), multipotent colonies can be distinguished from other colony types based on their appearance. In an independent experiment, after $6 \mathrm{~d}$ of culture, colonies predicted to be multipotent contained $289 \pm 237$ cells in $20 \%$ oxygen $(n=9)$ or $589 \pm 156$ cells in decreased oxygen $(n=6)$. This difference in colony size was statistically significant $(p=0.018)$. The colonies contained $7.1 \pm 5.6$ and $17.5 \pm 7.0$ dead cells per colony, respectively. These observations suggest that there is an increase in the size of NCSC colonies in decreased oxygen and that the increased size of colonies results primarily from an increased rate of proliferation rather than from reduced cell death. The increased proliferation of NCSCs in decreased oxygen culture is consistent with what has been observed with multipotent CNS progenitors (Studer et al., 2000) and skeletal muscle satellite cells (Csete and Wold, unpublished data).

NCSCs are instructed by BMP2 to differentiate into neurons (Shah et al., 1996; Morrison et al., 1999). As an independent test of neuronal potential, we added BMP2 to cultures of $\mathrm{p} 75^{+} \mathrm{P}_{0}{ }^{-}$cells in either decreased or $20 \%$ oxygen. After 4-6 d, the cultures were stained for peripherin to analyze the extent of neuronal differentiation. The results are shown in Table 2. As seen previously, a higher proportion of $\mathrm{p} 75^{+} \mathrm{P}_{0}{ }^{-}$cells survived and formed colonies in decreased oxygen, irrespective of whether BMP2 was added. Almost no colonies contained peripherin-positive (peripherin ${ }^{+}$) cells in the cultures that did not receive BMP2 (peripherin expression is normally not evident for at least $12 \mathrm{~d}$ under standard culture conditions). However, among cultures to which BMP2 was added, decreased oxygen was associated with a significantly higher proportion of colonies that contained only neurons and a significantly lower proportion of colonies that contained no neurons. In decreased oxygen, nearly $60 \%$ of colonies contained only neurons and almost $95 \%$ of colonies contained at least some neurons (Table 2). Thus, neuronal differentiation was significantly promoted in decreased oxygen culture. Because neurogenic capacity is a distinguishing characteristic of NCSCs isolated from the sciatic nerve (Morrison et al., 1999), these data suggest that up to $95 \%$ of p $75^{+} \mathrm{P}_{0}{ }^{-}$cells may be NCSCs.

\section{Decreased oxygen culture reveals that most or all NCSCs isolated from sciatic nerve have SA lineage potential}

SA lineage cells have not yet been demonstrated to differentiate directly from individual NCSCs in clonal culture, raising the pos- 


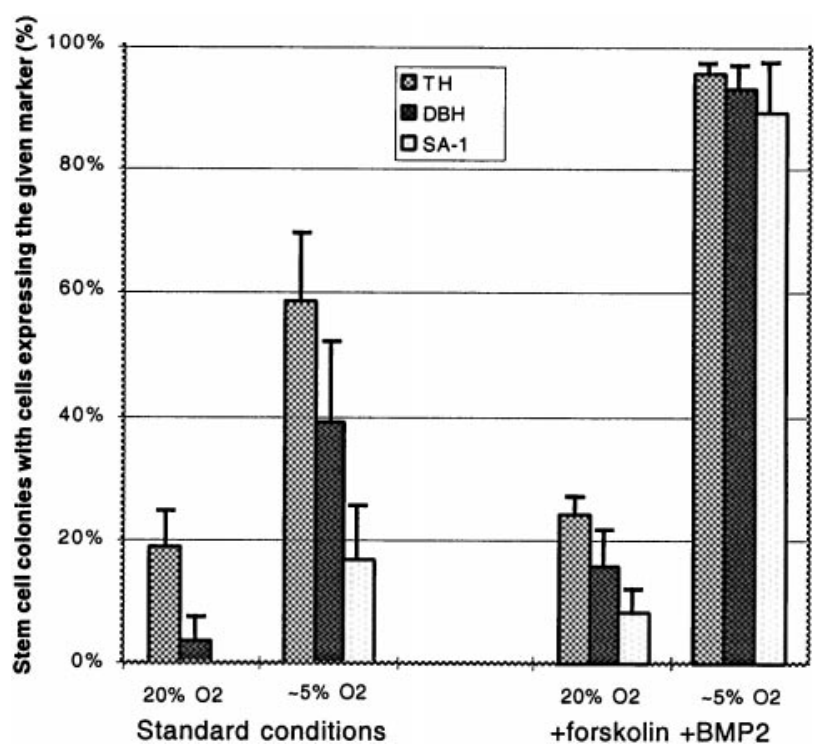

Figure 1. Decreased oxygen promotes sympathoadrenal lineage differentiation. Sciatic nerve $\mathrm{p} 75^{+} \mathrm{P}_{0}-$ cells were cultured in decreased oxygen or in $20 \%$ oxygen, for a total of $12 \mathrm{~d}$, either under standard conditions or with 5 $\mu \mathrm{M}$ forskolin and $1 \mathrm{ng} / \mathrm{ml} \mathrm{BMP} 2$ added after $6 \mathrm{~d}$ of standard culture. Cultures were fixed and stained with antibodies against TH, DBH, and the sympathoadrenal lineage marker SA-1. The bars show the percentage of stem cell colonies that contained any cells expressing the given markers. Error bars show SEs. Sympathetic markers were expressed at a significantly higher frequency in decreased oxygen and in the presence of forskolin and BMP2 $(p<0.05)$. In addition, in the presence of BMP2 plus forskolin, there were considerably more $\mathrm{TH}^{+}$cells per colony than in the absence of these factors (see Fig. $2 A, C$ ).

sibility that these stem cells lack catecholaminergic potential. Forskolin and BMP2 were shown previously to promote differentiation of $\mathrm{TH}^{+}$and $\mathrm{DBH}^{+}$cells in rat neural crest explants, but such differentiation was only observed in high-density cultures (Lo et al., 1999), leaving open the possibility that such SA lineage cells may have differentiated from restricted SA progenitors that survive only in mass cultures rather than from NCSCs. Given the beneficial effect of decreased oxygen on overall NCSC multilineage differentiation, we decided to test whether BMP2 plus forskolin would promote SA lineage differentiation by isolated NCSCs in clonal culture under these conditions.

p $75{ }^{+} \mathrm{P}_{0}{ }^{-}$cells were added to culture at clonal density in standard conditions in either decreased or $20 \%$ oxygen. After $6 \mathrm{~d}$ of culture, $5 \mu \mathrm{M}$ forskolin and $1 \mathrm{ng} / \mathrm{ml}$ BMP2 were added to some cultures, and the levels of CEE and FGF were concomitantly reduced to promote differentiation (see Materials and Methods). After a total of $12 \mathrm{~d}$ in culture, colonies were stained for three markers of sympathetic lineage differentiation: $\mathrm{TH}, \mathrm{DBH}$, and SA-1, a marker of chromaffin cells and sympathoadrenal progenitors (Carnahan and Patterson, 1991). In the absence of added forskolin and BMP2, decreased oxygen significantly increased the proportion of colonies that contained any cells expressing $\mathrm{TH}$, DBH, or SA-1 (Fig. 1), although the number of $\mathrm{TH}^{+}$cells in such colonies was relatively low (Fig. $2 C$ ). In decreased oxygen cultures supplemented with forskolin and BMP2, however, nearly all stem cell colonies contained $\mathrm{TH}^{+}, \mathrm{DBH}^{+}$and SA-1 ${ }^{+}$cells (Fig. 1), and the frequency of such cells within colonies as well as the intensity of marker expression was much higher (Fig. $2 A$ ). Individual cells grown in BMP2 plus forskolin in decreased $\mathrm{O}_{2}$ were seen to coexpress $\mathrm{TH}$ and $\mathrm{DBH}$ (Fig. 3A,C,E, arrowheads) or $\mathrm{TH}$ and SA-1 (antibodies against SA-1 and DBH are of the same isotype, so it was not possible to double stain for those markers) (Fig. $3 B, D, F$, arrowheads). These data therefore demonstrate that, under appropriate culture conditions, nearly all NCSCs isolated from E14.5 sciatic nerve exhibit SA lineage potential.

\section{$\mathrm{TH}^{+}$cells derived from NCSCs coexpress neuronal markers and release dopamine and norepinephrine}

Because SA-1 expression is normally downregulated during sympathetic neuron differentiation (Anderson et al., 1991), the fact that most $\mathrm{TH}^{+}$cells coexpressed SA-1 suggested that they might represent immature SA progenitors or SIF-like cells (Carnahan and Patterson, 1991). To promote overt neuronal differentiation by these cells, we cultured $\mathrm{p} 75^{+} \mathrm{P}_{0}{ }^{-}$cells in decreased oxygen at clonal density for $6 \mathrm{~d}$ in standard medium, followed by $6 \mathrm{~d}$ supplemented with forskolin and low BMP2, followed by a final $6 \mathrm{~d}$ supplemented by $50 \mathrm{ng} / \mathrm{ml}$ each of NGF and NT-3 (which have been shown to promote the differentiation and survival of sympathetic neuroblasts in vitro) (Birren et al., 1993; Verdi and Anderson, 1994).

Double-labeling of cultures grown under these conditions with antibodies to $\mathrm{TH}$ and neurofilament middle molecular weight subunit (NFM) revealed that the colonies contained three $\mathrm{TH}^{+}$cell types that were distinct with respect to morphology and NFM staining. One type displayed a polygonal morphology with small or absent neurites, very strong TH staining, and low NFM staining (Fig. 4A-C, arrow points), resembling SIF or chromaffin-like cells (Doupe et al., 1985b and references therein). There were also polygonal cells with longer varicose processes that had very strong TH staining and intermediate levels of NFM staining (Fig. 4B,E, arrowheads), appearing intermediate in phenotype between SIF cells and sympathetic neurons. Finally, other cells displayed round cell bodies, long neurites, a lower level of TH staining, and strong NFM staining, resembling immature sympathetic neurons (Fig. 4, arrows). Of 29 NCSC colonies cultured under neuron-promoting conditions and then double-labeled for TH and NFM, all contained at least some $\mathrm{TH}^{+}$cells resembling SIF cells or sympathetic neurons, although the SIF-like phenotypes appeared predominant. Under these conditions, $3.6 \pm 2.5 \%$ of cells in each colony were $\mathrm{TH}^{+}$. Because such stem cell colonies contain more than $10^{5}$ cells (Morrison et al., 1999), individual $\mathrm{p} 75^{+} \mathrm{P}_{0}{ }^{-}$cells therefore gave rise to thousands of SA lineage cells.

We used HPLC to determine whether colonies derived from individual NCSCs that contained $\mathrm{TH}^{+}$and $\mathrm{DBH}^{+}$cells also produced and/or secreted both DA and NE. Single p $75^{+} \mathrm{P}_{0}{ }^{-}$cells were sorted into individual wells of 96 well plates and then cultured under sympathetic neuron-promoting conditions. Some colonies were trypsinized, resuspended in $0.2 \mathrm{M}$ perchloric acid, and analyzed for DA and NE content by HPLC. DA and NE peaks were identified by retention times that were similar to authentic standards $(<0.1 \mathrm{~min})$. Three of three colonies contained DA, and two of three contained $\mathrm{NE}$; the $\mathrm{DA} / \mathrm{NE}$ molar ratio in colonies that contained both was $\sim 1: 1$. Other colonies were depolarized by incubation for $5 \mathrm{~min}$ in $40 \mathrm{~mm} \mathrm{KCl}$ in HBSS to induce transmitter release, and then the supernatants and the cells were analyzed by HPLC. Five of eight such supernatants contained DA, and six of eight contained $\mathrm{NE}$; in three of the five samples containing both transmitters, the ratio was close to $1: 3(\mathrm{DA} / \mathrm{NE})$. In cells extracted after depolarization, four of four contained some residual DA, but none contained detectable NE. These data are consistent with the fact that DA is both vesicular and cytoplasmic, whereas NE is exclusively vesicular in catecholaminergic cells.

\section{DISCUSSION}

Culturing NCSCs in a decreased oxygen environment promoted their survival, proliferation, multilineage differentiation, and revealed, for the first time, essentially uniform SA lineage capacity. Because $83 \%$ of clones exhibited multilineage differentiation in standard culture conditions and $95 \%$ of clones exhibited neuronal differentiation in response to BMP2, we estimate that the $\mathrm{p} 75^{+} \mathrm{P}_{0}{ }^{-}$ population is $83-95 \%$ pure for NCSCs. The ability of a decreased oxygen environment to promote survival and differentiation seemed to be preferential for stem cells in the conditions we studied, because other cell types present in the sciatic nerve were not similarly affected. The proliferation and survival of stem and progenitor cells from the CNS (Studer et al., 2000) and skeletal 

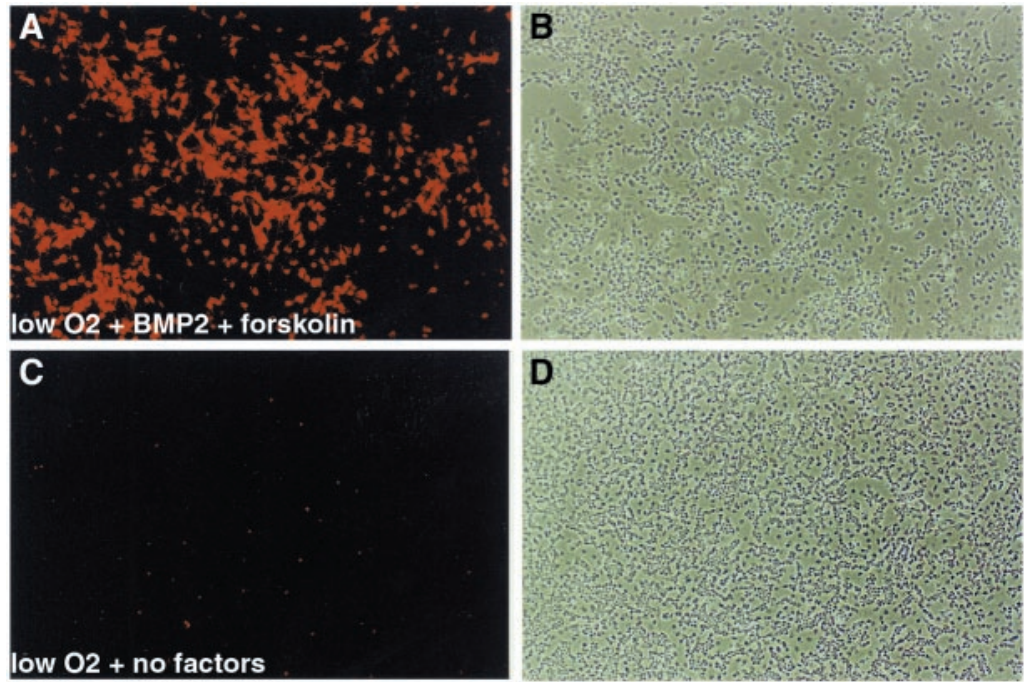

Figure 2. Induction of TH expression by culture in BMP2 plus forskolin in decreased oxygen. $A, C$, and $E$ are epifluorescence illumination of anti-TH antibody staining of the phase contrast fields shown in $B, D$, and $F$, respectively. $A, B$, A colony grown in decreased oxygen plus $1 \mathrm{ng} / \mathrm{ml}$ BMP 2 plus $5 \mu \mathrm{M}$ forskolin. $C$, $D$, A colony grown in decreased oxygen without added factors. $E, F$, A colony grown in $20 \%$ oxygen plus BMP2 plus forskolin. No TH expression was seen in cultures grown in $20 \%$ oxygen without BMP2 or forskolin in this experiment (data not shown). In general, only rare $\mathrm{TH}^{+}$cells were observed in $20 \%$ oxygen cultures. $A-F, 10 \times$ objective magnification.
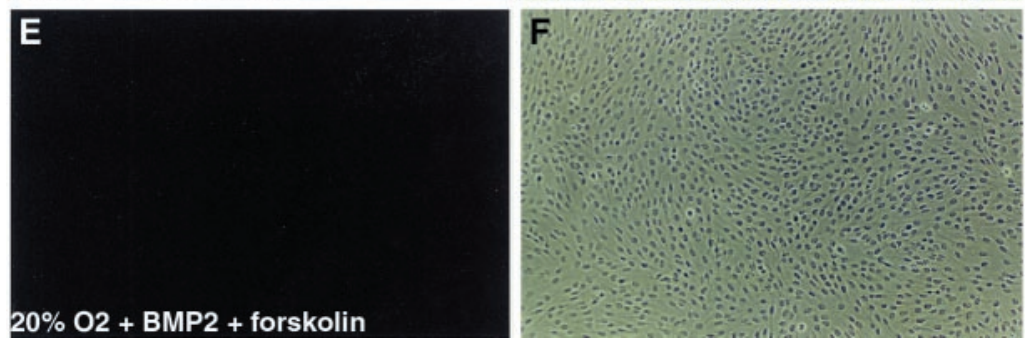
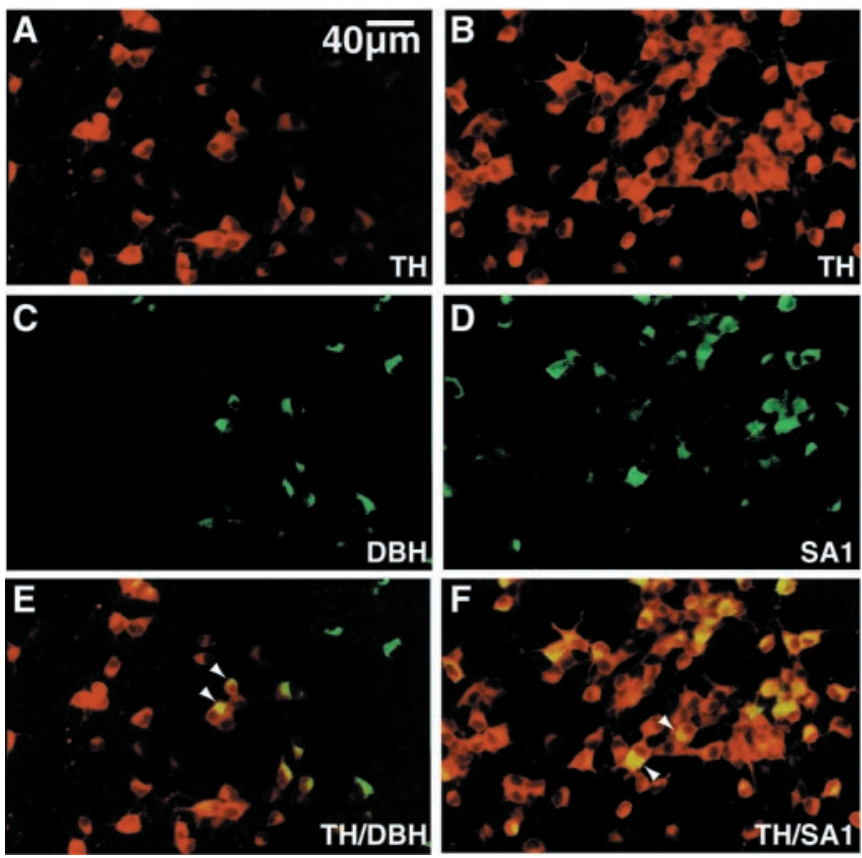

Figure 3. Coexpression of SA lineage markers. All panels are from cultures grown in decreased oxygen plus $1 \mathrm{ng} / \mathrm{ml} \mathrm{BMP} 2$ plus $5 \mu \mathrm{M}$ forskolin. Cultures were double-labeled with anti-TH $(A, B)$ and anti-DBH $(C)$ or anti-SA1 $(D)$. $E$ and $F$ show the merged images in $A, C$ and $B, D$, respectively. Thus, $A, C$, and $E$ represent the same field of one colony, and $B, D$, and $F$ represent the same field of a second colony. $C$ and $D$ were contrast-adjusted to subtract out background attributable to "bleed through" of R-phycoerythrin secondary antibody $(A, B)$ into the FITC channel $(C, D)$; this underestimates the number of $\mathrm{DBH}^{+}$and SA-1 ${ }^{+}$cells. Arrowheads in $E$ and $F$ indicate double-labeled cells. $A-F, 40 \times$ objective magnification.

muscle (Csete and Wold, unpublished data) are enhanced in decreased oxygen cultures as well.

Oxygen concentrations in culture of $<10 \%$ are often described as hypoxic because they are below atmospheric oxygen levels; however, dissolved oxygen levels in culture under such conditions remain high relative to oxygen concentrations in vivo, which average only 3\% (Guyton and Hall, 1996). Because the solubility of dissolved oxygen in interstitial fluid and culture medium is the same, a gas phase oxygen concentration of 3-6\% in culture should give rise to oxygen concentrations in the culture medium that approach average physiological levels. However, the precise relationship between the oxygen concentration we used in culture and oxygen concentrations in vivo is difficult to determine because oxygen levels vary widely in vivo. Moreover, the kinetics of oxygen diffusion and use may vary in important ways between the in vitro cultures and in vivo tissues. Thus, it is difficult to precisely compare oxygen levels in vitro and in vivo, but oxygen concentrations of 3-6\% in culture are much closer to physiological levels than the $20 \%$ oxygen used in most tissue culture experiments.

The mechanism by which reduced oxygen levels promote stem cell survival, proliferation, and differentiation is uncertain and probably complex. Fluctuations in physiological oxygen levels, as well as frank hypoxia, lead to profound physiological responses and changes in gene expression (Blancher and Harris, 1998). Decreased oxygen levels can induce pathways that promote survival, proliferation, and particular types of differentiation in various cell types; therefore, there may be a pathway that is induced by decreased oxygen levels that specifically promotes stem cell proliferation and survival. Alternatively, stem cells may be more sensitive to the toxic effects of oxidative stress in culture than mature cell types. Whatever the mechanism, decreased oxygen culture has the practical benefit of improving stem cell culture viability and differentiation, facilitating further studies of their biology.

Our data demonstrate that essentially all isolated NCSCs have the capacity to generate SA lineage cells in culture. Whether these stem cells can generate other kinds of PNS neurons will require further study. Interestingly, the catecholaminergic differentiation of CNS stem cells is also promoted by decreased oxygen culture, although in that case the cells were dopaminergic but not noradrenergic (Studer et al., 2000). In PNS cells studied in vitro, TH is thought to be upregulated by reduced oxygen via both transcriptional and post-transcriptional mechanisms (Czyzyk-Krzeska et al., 
Colony 1 TH/NFM
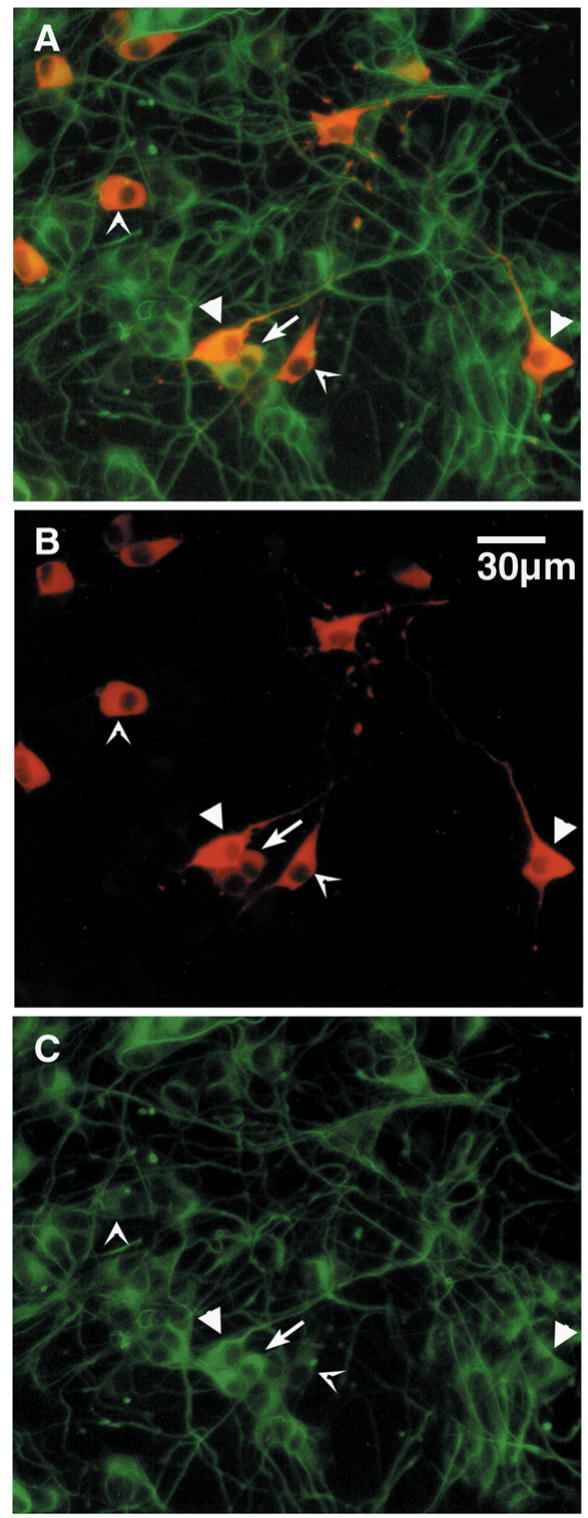

Colony 2 TH/NFM
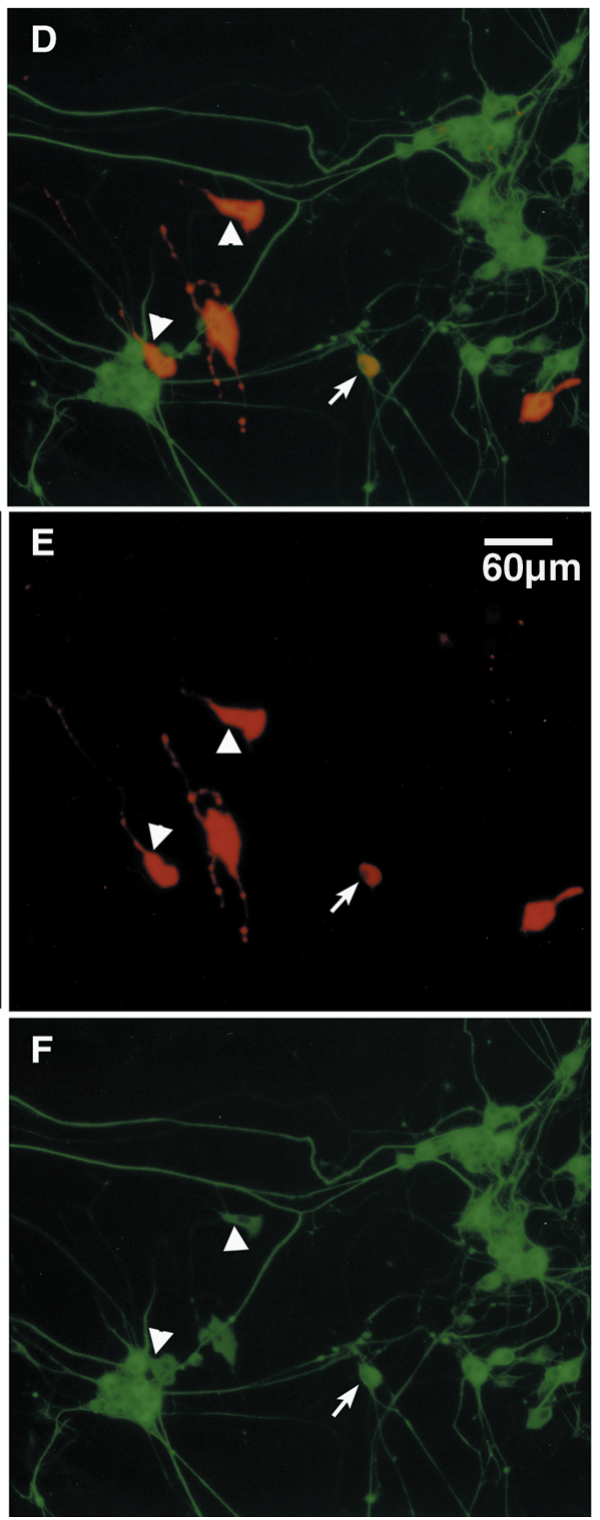

Figure 4. Neuronal differentiation of SA lineage cells. NFM ( green) and TH (red) staining of stem cell colonies cultured from $\mathrm{p} 75^{+} \mathrm{P}_{0}{ }^{-}$cells in decreased oxygen in the presence of BMP2 plus forskolin, followed by additional growth in NT-3 plus NGF. $A-C$ show the same field of a single colony photographed with a $40 \times$ objective. $D-F$ show one field of a different colony photographed with a $20 \times$ objective. $A$ and $D$ show superimpositions of the NFM and TH staining; $B$ and $E$ show tyrosine hydroxylase staining; $C$ and $F$ show NFM staining. Arrow points $(A-C)$ indicate SIF-like cells with polygonal morphology, short or absent neurites, high levels of TH staining, and low levels of NFM staining. Arrowheads indicate cells that appear transitional between SIF-like cells and sympathetic neurons, with polygonal morphology, long neurites, high levels of TH staining, and high NFM staining. Arrows indicate cells that resemble sympathetic neurons, with rounded cell bodies, long neurites, moderate TH staining, and high NFM staining.
1992, 1994; Paulding and Czyzyk-Krzeska, 1999). Oxygen regulation of DBH expression in vitro is less well studied, although maternal hypoxia may lead to upregulation of fetal adrenal DBH (Holgert et al., 1995). In all of these previous studies, however, the levels of expression of these enzymes were only examined in already differentiated catecholaminergic cells (Czyzyk-Krzeska et al., 1992, 1994; Paulding and Czyzyk-Krzeska, 1999). Our results suggest that commitment of multipotent stem cells to the SA lineage may also be promoted by reduced oxygen in vitro, although it remains possible that decreased oxygen additionally promoted the survival of SA lineage cells.

There is evidence to suggest that decreased oxygen may be important for the differentiation of SA derivatives in vivo as well as in vitro. Recently, EPAS1, a bHLH-PAS domain transcription factor that binds to an essential hypoxia-responsive element in the TH promoter (Norris and Millhorn, 1995; Millhorn et al., 1997), was shown to be required for the development of noradrenergic cells in vivo (Tian et al., 1998). Although the mechanism by which the EPAS1 mutation affects catecholaminergic differentiation was not investigated, these genetic data suggest that the effect of decreased oxygen to enhance TH expression by NCSCs in vitro may be mediated by EPAS1. By the same token, our in vitro results suggest that the EPAS1 knock-out phenotype may reflect an im- portant role for hypoxia-inducible pathways in SA lineage specification or differentiation in vivo.

Irrespective of the cellular and molecular mechanisms involved, the finding that decreased oxygen culture can promote the production of greatly expanded numbers of dopaminergic neurons from individual, purified stem cells may provide an important tool to facilitate transplantation into Parkinson's patients. Under our culture conditions, individual NCSCs gave rise to an average of $>3000 \mathrm{TH}^{+}$cells per colony. This was without any special effort to promote stem cell expansion or to optimize sympathetic differentiation. It seems likely that modest efforts in those regards could yield additional substantial increases in the numbers of dopaminergic cells. The ability to generate thousands or millions of dopaminergic cells from a single stem cell could provide both a defined precursor cell and adequate numbers of dopaminergic cells for therapeutic transplantation.

\section{REFERENCES}

Anderson DJ (1993) Molecular control of cell fate in the neural crest: the sympathoadrenal lineage. Annu Rev Neurosci 16:129-158.

Anderson DJ, Carnahan J, Michelsohn A, Patterson PH (1991) Antibody markers identify a common progenitor to sympathetic neurons and chromaffin cells in vivo, and reveal the timing of commitment to neuronal differentiation in the sympathoadrenal lineage. J Neurosci 11:3507-3519. 
Birren SJ, Lo LC, Anderson DJ (1993) Sympathetic neurons undergo a developmental switch in trophic dependence. Development 119:597-610. Bjorklund A (1993) Better cells for brain repair. Nature 362:414-415.

Blancher C, Harris AL (1998) The molecular basis of the hypoxia response pathway: tumor hypoxia as a therapy target. Cancer Metastasis Rev 17:187-194.

Carnahan JF, Patterson PH (1991) Generation of monoclonal antibodies that bind preferentially to adrenal chromaffin cells and the cells of embryonic sympathetic ganglia. J Neurosci 11:3493-3506.

Czyzyk-Krzeska MF, Bayliss DA, Lawson EE, Millhorn DE (1992) Regulation of tyrosine hydroxylase gene expression in the rat carotid body by hypoxia. J Neurochem 58:1538-1546.

Czyzyk-Krzeska MF, Furnari BA, Lawson EE, Millhorn DE (1994) Hypoxia increases the rate of transcription and stability of tyrosine hydroxylase mRNA in pheochromocytoma (PC12) cells. J Biol Chem 269:760-764.

Doupe AJ, Landis SC, Patterson PH (1985a) Environmental influences in the development of neural crest derivatives: glucocorticoids, growth factors and chromaffin cell plasticity. J Neurosci 5:2119-2142.

Doupe AJ, Patterson PH, Landis SC (1985b) Small intensely fluorescent (SIF) cells in culture: role of glucocorticoids and growth factors in their development and phenotypic interconversions with other neural crest derivatives. J Neurosci 5:2143-2160.

Fraser SE, Bronner-Fraser ME (1991) Migrating neural crest cells in the trunk of the avian embryo are multipotent. Development 112:913-920.

Freed CR, Breeze RE, Rosenberg NL, Schneck SA, Kreik E, Qi J-X, Lone T, Zhang Y-B, Snyder JA, Wells TH, Ramig LO, Thompson L, Mazziotta JC, Huang SC, Grafton ST, Brooks D, Sawle G, Schroter G, Ansar AA (1992) Survival of implanted fetal dopamine cells and neurologic improvement 12 to 46 months after transplantation for Parkinson's disease. N Engl J Med 327:1549-1555.

Guyton AC, Hall JE (1996) Transport of oxygen and carbon dioxide in the blood and body fluids. In: Textbook of medical physiology, pp 513-523. Philadelphia: Saunders.

Holgert H, Pequignot JM, Lagercrantz H, Hokfelt T (1995) Birth-related up-regulation of mRNA encoding tyrosine hydroxylase, dopamine-betahydroxylase, neuropeptide tyrosine, and prepro-enkephalin in rat adrenal medulla is dependent on postnatal oxygenation. Pediat Res 37:701-706.

Jessen KR, Mirsky R (1999) Schwann cells and their precursors emerge as major regulators of nerve development. Trends Neurosci 22:402-410.

Lo L, Morin X, Brunet J-F, Anderson DJ (1999) Specification of neurotransmitter identity by Phox 2 proteins in neural crest stem cells. Neuron 22:693-705.

Luquin MR, Montoro RJ, Guillen J, Saldise L, Insausti R, Rio JD, LopezBarneo J (1999) Recovery of chronic parkinsonian monkeys by autotransplants of carotid body cell aggregates into putamen. Neuron 22:743-750.
Millhorn DE, Raymond R, Conforti L, Zhu W, Beitner-Johnson D, Filisko T, Genter MB, Kobayashi S, Peng M (1997) Regulation of gene expression for tyrosine hydroxylase in oxygen sensitive cells by hypoxia. Kidney Int 51:527-535.

Morrison SJ, White PM, Zock C, Anderson DJ (1999) Prospective identification, isolation by flow cytometry, and in vivo self-renewal of multipotent mammalian neural crest stem cells. Cell 96:737-749.

Norris ML, Millhorn DE (1995) Hypoxia-induced protein-binding to O2responsive sequences on the tyrosine hydroxylase gene. J Biol Chem 270:23774-23779.

Paulding WR, Czyzyk-Krzeska MF (1999) Regulation of tyrosine hydroxylase mRNA stability by protein-binding, pyrimidine-rich sequence in the 3'-untranslated region. J Biol Chem 274:2532-2538.

Reissman E, Ernsberger U, Francis-West PH, Rueger D, Brickell PD, Rohrer H (1996) Involvement of bone morphogenetic protein-4 and bone morphogenetic protein-7 in the differentiation of the adrenergic phenotype in developing sympathetic neurons. Development 122:2079-2088.

Shah NM, Groves A, Anderson DJ (1996) Alternative neural crest cell fates are instructively promoted by TGF $\beta$ superfamily members. Cell 85:331-343.

Stemple DL, Anderson DJ (1992) Isolation of a stem cell for neurons and glia from the mammalian neural crest. Cell 71:973-985.

Studer L, Tabar V, McKay RDG (1998) Transplantation of expanded mesencephalic precursors leads to recovery in parkinsonian rats. Nat Neurosci 1:290-295.

Studer L, Csete M, Lee S-H, Kabbani N, Walikonis J, Wold B, McKay R (2000) Enhanced proliferation, survival, and dopaminergic differentiation of CNS precursors in lowered oxygen. J Neurosci 20:7377-7383.

Tian H, Hammer RE, Matsumoto AM, Russell DW, McKnight SL (1998) The hypoxia-responsive transcription factor EPAS1 is essential for catecholamine homeostasis and protection against heart failure during embryonic development. Genes Dev 12:3320-3324.

Varley JE, Maxwell GD (1996) BMP-2 and BMP-4, but not BMP-6, increase the number of adrenergic cells which develop in quail trunk neural crest cultures. Exp Neurol 140:84-94.

Varley JE, Wehby RG, Rueger DC, Maxwell GD (1995) Number of adrenergic and islet-1 immunoreactive cells is increased in avian trunk neural crest cultures in the presence of human recombinant osteogenic protein-1. Dev Dyn 203:434-447.

Verdi JM, Anderson DJ (1994) Neurotrophins regulate sequential changes in neurotrophin receptor expression by sympathetic neuroblasts. Neuron 13:1359-1372.

Zawada WM, Cibelli JB, Choi PK, Clarkson ED, Golueke PJ, Witta SE, Bell KP, Kane J, Ponce de Leon FA, Jerry DJ, Robl JM, Freed CR, Stice SL (1998) Somatic cell cloned transgenic bovine neurons for transplantation in parkinsonian rats. Nat Med 4:569-574. 